\title{
Beïda Chikhi, Les romans d'Assia Djebar
}

\section{Giuliva Milò}

\section{(2) OpenEdition}

\section{Journals}

\section{Édition électronique}

URL : http://journals.openedition.org/studifrancesi/36787

DOI : 10.4000/studifrancesi.36787

ISSN : 2427-5856

\section{Éditeur}

Rosenberg \& Sellier

\section{Édition imprimée}

Date de publication : 1 juillet 2005

Pagination : 206-207

ISSN : 0039-2944

\section{Référence électronique}

Giuliva Milò, «Beïda Chikhi, Les romans d'Assia Djebar », Studi Francesi [En ligne], 145 (XLIX | I) | 2005,

mis en ligne le 30 novembre 2015, consulté le 18 avril 2021. URL : http://journals.openedition.org/ studifrancesi/36787 ; DOI : https://doi.org/10.4000/studifrancesi.36787

\section{Ce document a été généré automatiquement le 18 avril 2021.}

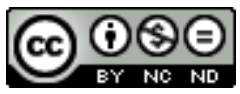

Studi Francesi è distribuita con Licenza Creative Commons Attribuzione - Non commerciale - Non opere derivate 4.0 Internazionale. 


\title{
Beïda Chikhi, Les romans d'Assia Djebar
}

\author{
Giuliva Milò
}

\section{RÉFÉRENCE}

BË̈DA CHIKHI, Les romans d'Assia Djebar, Alger, Office des Publications Universitaires, 2002, pp. 251.

1 En 1989 Beïda Chikhi, alors professeur à l'université d'Alger, dédiait un petit ouvrage de 130 pages à l'étude des romans d'Assia Djebar, encore très peu connue du grand public. Pour contribuer à ce qui se tisse constamment entre la France et l'Algérie, c'est avec enthousiasme qu'elle a répondu à l'appel de l'OPU pour cette réédition du livre où elle apporte le complément de lectures, d'expériences et de vécu accumulés au fil du temps. Ainsi la première lecture est refondue dans cette nouvelle version où l'analyse se prolonge jusqu'à La femme sans sépulture (2002), avec un intérêt soutenu pour l'écriture djebarienne, qui se veut essentielle dans la création littéraire à la fois comme art de réussir les nuances et objet de recherche sémiologique. L'analyse veut restituer à la production djebarienne toute sa complexité à travers un itinéraire qui, à partir des différents réseaux sémantiques et esthétiques se faisant écho d'une œuvre à l'autre, décrypte les signes qui indiquent les préoccupations majeures liées à une réalité fortement ancrée dans l'humus socio-historique algérien. Ce faisant, Beïda Chikhi semble répondre à certains partisans d'une herméneutique absconse dont l'intérêt s'est surtout porté sur le pur savoir-faire linguistique de l'auteur, amputant ainsi sa production d'un référent dont on ne peut faire abstraction. Elle réussit à montrer comment Assia Djebar, à la fois artiste et historienne, met en œuvre tous ses savoirs dans une œuvre où "les figures surprenantes surgies par hasard des mailles du langage disent le brûlant d'accéder à la perfection de la représentation esthétique" sans, toutefois, jamais cesser de marquer un engagement qui, comme dirait Umberto Eco, oblige le lecteur à garder constamment un œil fixé sur "l'intention du texte". 


d

dans le n. $1086 \mathrm{du}$ «Nouvel Observateur» faisait remarquer le souffle nouveau que la
langue de L'amour, la fantasia apportait au français; l'autre, le discours prononcé par Assia Djebar, consacrée écrivain international, lors de la remise du prix de la paix de Francfort le 22 octobre 2000. Le premier chapitre s'attache à voir comment le fait historique vient s'inscrire, avec plus ou moins de force, au cœur de la fiction en cueillant dans les romans examinés une épaisseur où tout prend valeur de signe "dans un univers de formes qui rappellent les constructions de base propres aux sémioticiens". Le critique décèle clairement l'objectif principal de l'auteur, qui est celui de faire participer la littérature au conflit d'interprétation de l'Histoire, et analyse la manière dont l'appareil sémiotique des romans djebariens prend en charge tout ce qui dans l'Histoire est resté inexpliqué. Beïda Chikhi illustre comment la fiction djebarienne s'installe dans les failles des systèmes officiels en prenant comme sujet essentiel tout ce que les discours dominants ne mentionnent pas, à savoir le "refoulé" et le "marginalisé" en tant que véritables enjeux historiques et politiques. C'est bien dans cette optique qu'est lu, dans le chapitre suivant, le roman féminin algérien pour lequel celui d'Assia Djebar constitue un modèle. Ce qui est surtout mis en valeur, c'est la structure duelle de l'univers symbolique, structure produite par des lectures contradictoires de l'Histoire émanant, d'une part, du sujet à la recherche de son bonheur, de l'autre, du corps social soucieux de la préservation d'une "loi révélée mais non écrite". L'analyse s'attarde sur l'élaboration de ce conflit, présent dès les premiers textes et poussé à son plus haut degré à travers la problématique du regard féminin forgé par les écrans du voile et des interdits sociaux. Pour Chikhi, l'œil est le lieu à partir duquel s'organise la dimension critique de la production de l'auteur. Le texte djebarien est à lire, à écouter, à voir; c'est pourquoi, dit-elle, il met en œuvre deux médiations esthétiques essentielles, l'une picturale, l'autre musicale. Sont ainsi isolés dans L'amour, la fantasia (1985) et Femmes d'Alger dans leur appartement (1980) les passages où la fantaisie esthétique vient faire bouger le texte ou/et dialogue avec les tableaux de Delacroix ou de Picasso. Le recours à la médiation plastique s'impose au lecteur à travers un geste pictural qui fixe à jamais l'histoire algérienne. Assia Djebar emprunte à la peinture dans ses multiples expressions (esquisse, aquarelle, lithographie) et au cinéma tout ce qui peut féconder son écriture, tout ce qui peut renforcer le regard critique et la mémoire. Le critique étudie les significations profondes qui émergent des parallélismes établis entre les différents langages, musical, pictural, narratifs, des similitudes opérées entre les traditions musicales du Maghreb et l'enchaînement des plans musicaux des compositions occidentales. Le romanesque fait usage des genres musicaux appropriés à des situations spécifiques, mais les rythmes et les sonorités s'imposent toujours dans l'intervalle de leur signification car ce qui produit l'émotion et rend le lecteur réceptif à la magie, nous dit Beïda Chikhi, c'est la tension perpétuelle, le contrepoint continu entre le son et le sens.

5 L'essayiste réfléchit encore sur la façon dont les textes spécifiquement féminins, à savoir, Ombre Sultane (1987), La femme en morceaux (dans Oran, langue morte, 1997) et Loin de Médine (1991), participent à la mise en cause du fondement unitaire de la pensée arabe en général, maghrébine en particulier. Ils explicitent ce mouvement de passage

Studi Francesi, 145 (XLIX | I) | 2005 
vers la transgression, engageant l'être-femme dans la mise en récit de ses propres fantasmes comme fictions libératrices. La fiction entame progressivement le réel car elle se fait investigation, quête de rationalité et imagine des situations qui aident à dévoiler des stratégies de changement. Si Les nuits de Strasbourg (1997) a souvent été considéré un roman à part dans la production djebarienne, notre critique tend en revanche à l'englober dans l'unité de l'œuvre en mettant en relief les moments forts de l'Histoire où la ville acquiert des significations insoupçonnées. Elle s'attache à découvrir, à travers le filigrane de l'écriture, le sens du choix d'un contexte strasbourgeois: les rapprochements entre la destinée d'Alger dans les autres romans et celle de Strasbourg deviennent inévitables. Des parallélismes se tissent aussi entre l'histoire de la ville et l'histoire personnelle de l'héroïne hantée par les mêmes fantômes que la narratrice de Vaste est la prison (1995). Le dernier chapitre est entièrement consacré à la question linguistique qui, chez Djebar, donne naissance à l'une des plus belles illustrations de la notion de corps-texte. L'imaginaire de la navigation linguistique restitue dans les figures de style et les scénographies corporelles, un cheminement charnel et sensuel. Qui mieux que Beïda Chikhi, experte en langues et cultures maghrébines, aurait pu deviner les multiples balises du texte? Balises qui rappellent le lien vital entre la langue, la mémoire, le corps. Qui mieux qu'elle pouvait signaler au lecteur les corpus linguistiques où les traces de la langue maternelle s'effacent peu à peu devant le français? Elle nous introduit dans un discours romanesque qui, à la suite des grands écrivains algériens tels que Kateb Yacine, Mohamed Dib ou Mouloud Mammeri, mise sur le pouvoir de la langue et sur le sens refoulé de la parole. En dénouant le pacte social fondé sur l'interdit, il restaure le corps dans sa langue de liberté. Ainsi, d'une œuvre à l'autre, le texte djebarien formule le désir de vie de la langue du corps désirant. La langue du corps, effacée puis retrouvée, accède au symbole; elle incarne toutes les langues perdues ou refoulées et finalise le rapport entre écriture et symbolisation. Ceci explique le point de vue psychanalytique qu'autorise aussi la lecture de la nouvelle La femme en morceaux (dans Oran, langue morte), Loin de Médine ou La femme sans sépulture. Ce dernier roman permet de conclure sur le thème de l'expatriation et le vibrant désir de faire revivre, à travers l'écriture, l'espace de l'origine. Le retour sur les lieux d'un bonheur passé, à réinventer, participe de ce refus de l'exil commun à tous les écrivains algériens en quête d'un nouveau lieu. 